\title{
Central retinal artery and vein collapse pressure in giant cell arteritis versus nonarteritic anterior ischaemic optic neuropathy
}

\begin{abstract}
Arteritic anterior ischaemic optic neuropathy and nonarteritic anterior ischaemic optic neuropathy are acute optic neuropathies, which have to be differentiated from each other. It was the purpose of this study to assess whether ophthalmodynamometry with an assessment of the collapse pressure of the central retinal artery (CRA) and vein (CRV) is helpful for that. Using a Goldmann contact lens-associated ophthalmodynamometer, the diastolic collapse pressure of the CRA and CRV were measured in six patients (eight eyes) with giant cell arteritis-induced anterior ischaemic optic neuropathy (GC-AION) and in 10 patients (12 eyes) with acute non-arteritic anterior ischaemic optic neuropathy (NAION). CRA collapse pressure was significantly $(P=0.001 ; 95 \%$ confidence interval $(\mathrm{CI}):-68.7$,

-20.0) lower in the GC-AION group $(52.7 \pm 24.6$ arbitrary units) than in the NAION group $(\mathbf{9 7 . 0} \pm \mathbf{2 5 . 8}$ arbitrary units). CRV collapse pressure did not vary significantly $(P=0.47)$. As measured by ophthalmodynamometry, CRA pressure is significantly lower in GC-AION than in NAION. CRV pressure does not vary markedly. These finding may be helpful for the clinical differentiation between GC-AION and NAION, and may give hints for the pathogenesis.
\end{abstract}

Department of

Ophthalmology, Faculty of Clinical Medicine Mannheim, Ruprecht-Karls-University of Heidelberg, Mannheim, Germany

Correspondence: JB Jonas, Universitäts-Augenklinik, Theodor-Kutzer-Ufer 1-3, Mannheim 68167, Germany Tel: + 49621383 2652; Fax: + 496213833803 . E-mail: Jost.Jonas@ augen.ma.uni-heidelberg.de

Received: 24 October 2006 Accepted in revised form: 16 February 2007 Published online: 23 March 2007

Proprietary interest: none
Eye (2008) 22, 556-558; doi:10.1038/sj.eye.6702792; published online 23 March 2007

Keywords: ophthalmodynamometry; anterior ischaemic optic neuropathy; giant cell arteritis; central retinal artery; central retinal vein
JB Jonas and B Harder

\section{Introduction}

Giant cell arteritis-induced anterior ischaemic optic neuropathy (GC-AION) and non-arteritic anterior ischaemic optic neuropathy (NAION) are entities, which are both characterized by an acute swelling of the optic nerve head and sudden loss of vision. ${ }^{1}$ As the differentiation between both diseases is clinically important with regard to marked differences in therapy and prognosis and because both entities affect the vascular system, it was the purpose of the present study to assess whether the ophthalmodynamometrically assessable pressure in the central retinal artery and vein differs between both diseases. The results could be helpful for the clinical differentiation between both diseases and could give hints for the pathogenesis.

\section{Methods}

The clinical observational comparative case series study included six patients (8 eyes) with GC-AION and 10 patients (12 eyes) with NAION. The University Ethics Committee had approved that the methods applied in the study adhered to the tenets of the declaration of Helsinki for the use of human subjects in biomedical research. In the GC-AION group, the diagnosis was based on the clinical symptoms with a sudden and marked loss in vision, headaches often persisting for weeks and months, undetermined pain in the back and shoulder, a markedly elevated erythrocyte sedimentation rate, an elevated C-reactive protein level in the serum and a histological biopsy of the superficial temporal artery showing characteristic giant cell arteritis. In the 
NAION group, the erythrocyte sedimentation rate and the level of C-reactive protein were within the age-related normal limits, and biopsies had shown no histological signs of giant cell arteritis. Clinically, patients of the NAION group reported about loss in vision, often noticed in the morning, without headache or unexplained weight loss in the preceding months. The erythrocyte sedimentation rate and the level of the $\mathrm{C}$-reactive protein were within the age-related normal limits. There was no history of marked loss in body weight in the previous 2 months nor of marked pain in the shoulder or head.

For all patients included, ophthalmodynamometry was performed. After medical mydriasis, the optic nerve head was visualized using a non-contact ophthalmoscopic lens to assess a spontaneous pulsation of the central retinal vein. In the second step, a new ophthalmodynamometer (Meditron, D-66333 Völklingen, Germany) was used consisting of a conventional Goldmann contact lens fitted with a pressure sensor ring at its outer margin where the lens is usually held during an ophthalmoscopic examination. The lens was placed onto the corneal surface with topical anaesthesia. Slightly applying increasing pressure onto the contact lens, the optic nerve head was continuously observed

biomicroscopically. When the central retinal artery or vein or their branches on the surface of the optic nerve head showed early pulsations, the pressure asserted onto the lens and measured by the ophthalmodynamometer in arbitrary units was noted. All measurements were repeated nine times. The mean of the 10 measurements was taken for further statistical analysis. The reproducibility of the new ophthalmodynamometric technique examined in a previous study was $9.1 \pm 4.2 \%$

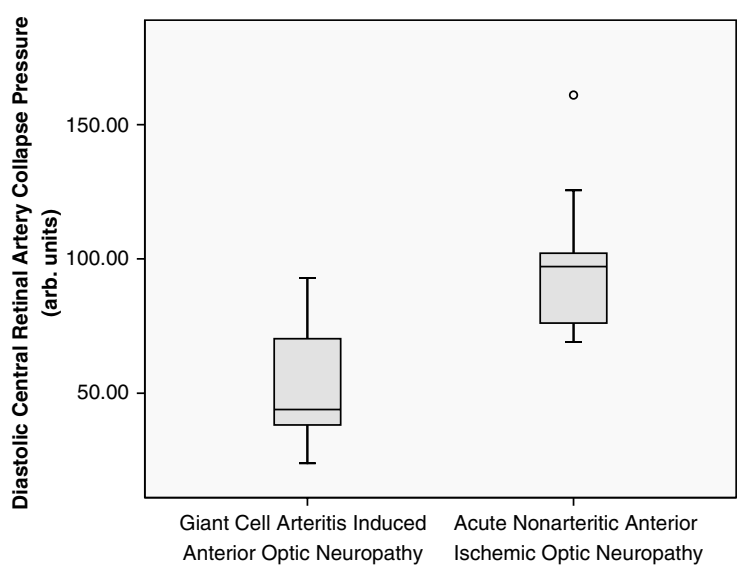

Figure 1 Box plot showing the statistically significant difference $(P<0.001)$ in the distribution of diastolic central retinal artery collapse pressure as measured by ophthalmodynamometry in eight eyes with giant cell arteritis induced acute optic neuropathy versus 12 eyes with acute non-arteritic anterior ischaemic optic neuropathy.

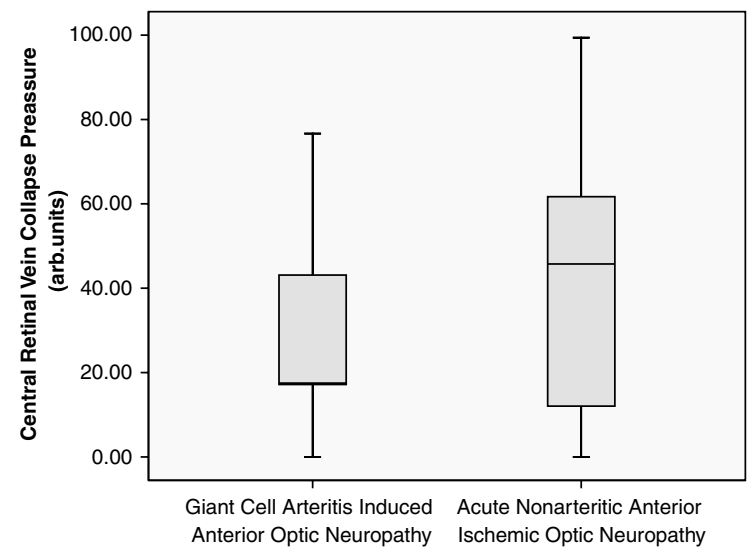

Figure 2 Box plot showing the distribution of diastolic central vein collapse pressure as measured by ophthalmodynamometry in eight eyes with giant cell arteritis-induced acute optic neuropathy versus 12 eyes with acute non-arteritic anterior ischaemic optic neuropathy, with no statistically significant $(P=0.47)$ difference between the two groups.

for re-determinations of the diastolic central retinal artery collapse pressure, and $15.9 \pm 11.9 \%$ for re-determinations of the diastolic central retinal vein collapse pressure. ${ }^{2}$ The method has already been described in detail. ${ }^{2,3}$

\section{Results}

Central retinal artery collapse pressure was significantly $(P=0.001 ; 95 \%$ confidence interval $(\mathrm{CI}):-68.7,-20.0)$ lower in the GC-AION group $(52.7 \pm 24.6$ arbitrary units) than in the NAION (97.0 \pm 25.8 arbitrary units). Central retinal vein collapse pressure did not vary significantly $(P=0.47 ; 95 \% \mathrm{CI}:-51.1,25.6)$ between the GC-AION group $(30.9 \pm 29.9$ arbitrary units) and the NAION $(43.7 \pm 34.9$ arbitrary units) (Figures 1 and 2).

\section{Conclusion}

As measured by a new ophthalmodynamometer with direct visualization of the central retinal vessels during the ophthalmodynamometric examination, the results of the present study suggest that the central retinal artery pressure is lower in patients with GC-AION than in those with acute NAION (Figure 1). The finding may be used clinically to help in the differentiation between both diseases. Pathogenically, it may reflect that in GC-AION, the retinal arteries, in addition to the short posterior ciliary arteries, can be affected by the inflammatory process. The study may add to the clinical application of modern ophthalmodynamometry as already demonstrated in previous investigations on glaucoma, measurement of intracerebral pressure, assessment of tissue pressure in the orbit in patients with endocrine 
orbitopathy, ischaemic ophthalmopathy and retinal vein occlusions. $^{4-12}$

\section{References}

1 Hayreh SS, Podhajsky PA, Zimmerman B. Ocular manifestations of giant cell arteritis. Am J Ophthalmol 1998; 125: 509-520.

2 Jonas JB. Reproducibility of ophthalmodynamometric measurements of the central retinal artery and vein collapse pressure. Br J Ophthalmol 2003; 87: 577-579.

3 Jonas JB. Central retinal artery and vein pressure in patients with chronic open-angle glaucoma. Br J Ophthalmol 2003; 87: 949-951.

4 Morgan WH, Balaratnasingam C, Hazelton ML, House PH, Cringle SJ, Yu DY. The force required to induce hemivein pulsation is associated with the site of maximum field loss in glaucoma. Invest Ophthalmol Vis Sci 2005; 46: 1307-1312.

5 Balaratnasingam C, Morgan $\mathrm{WH}$, Hazelton ML, House PH, Bany CJ, Chan $\mathrm{H}$ et al. Value of retinal vein pulsation characteristics in predicting increased optic disc excavation. Br J Ophthalmol 2006 Oct 11; [E-pub ahead of print].

6 Querfurth HW, Arms SW, Lichy CM, Irwin WT, Steiner T. Prediction of intracranial pressure from noninvasive transocular venous and arterial hemodynamic measurements: a pilot study. Neurocrit Care 2004; 1: 183-194.

7 Meyer-Schwickerath R, Stodtmeister R, Hartmann K. Non-invasive determination of intracranial pressure. (Physiological basis and practical procedure). Klin Monatsbl Augenheilkd 2004; 221: 1007-1011.

8 Morgan WH, Hazelton ML, Azar SL, House PH, Yu DY, Cringle SJ et al. Retinal venous pulsation in glaucoma and glaucoma suspects. Ophthalmology 2004; 111: 1489-1494.

9 Motschmann H, Muller C, Kuchenbecker J, Walter S, Schmitz K, Schutze M et al. Ophthalmodynamometry: a reliable method for measuring intracranial pressure. Strabismus 2001; 9: 13-16.

10 Firsching R, Schutze M, Motschmann H, Behrens-Baumann W. Venous opthalmodynamometry: a noninvasive method for assessment of intracranial pressure. J Neurosurg 2000; 93: 33-36.

11 Draeger J, Rumberger E, Hechler B. Intracranial pressure in microgravity conditions: non-invasive assessment by ophthalmodynamometry. Aviat Space Environ Med 1999; 70: 1227-1229.

12 Morgan WH, Yu DY, Alder VA, Cringle SJ, Constable IJ. Relation between pressure determined by ophthalmodynamometry and aortic pressure in the dog. $\mathrm{Br} \mathrm{J}$ Ophthalmol 1998; 82: 821-825. 